\title{
A Case of Right Apogeotropic Posterior Semicircular Canal BPPV that Initially Emulated as Left Anterior Semicircular Canal BPPV
}

Ajay Kumar Vats ${ }^{1}$ Sudhir Kothari ${ }^{2}$ Anirban Biswas ${ }^{3}$

\author{
${ }^{1}$ Division of Medicine, Department of Neurology, Chaudhary \\ Hospital and Medical Research Centre Private Limited, \\ Udaipur, Rajasthan, India \\ ${ }^{2}$ Department of Neurology, Poona Hospital and Research Centre, \\ Pune, Maharashtra, India \\ ${ }^{3}$ Vertigo and Deafness Clinic, Institute of Neurotology, Kolkata, \\ West Bengal, India
}

Ann Otol Neurotol:2021;3:93-99

\begin{abstract}
Address for correspondence Ajay Kumar Vats, MBBS, MD, DM, Division of Medicine, Department of Neurology, Chaudhary Hospital and Medical Research Centre Private Limited, 472-473, Sector 4, Hiran Magri, Udaipur, Rajasthan 313002, India (e-mail:vatsneuro@gmail.com).
\end{abstract}

\section{Abstract}

Keywords

- apo-PSC-BPPV

- geo-PSC-BPPV

- Dix-Hallpike test

- ampullopetal

- ampullofugal

- downbeating

nystagmus
In any patient with a history of rotational vertigo triggered by changes in the position of head relative to the gravity, whose oculomotor patterns elicit a positional downbeating nystagmus ( $p$-DBN), the localization could be either central in the brainstem, midline cerebellum, or at the craniocerebral junction; or else peripheral due to one of the rare variants of benign paroxysmal positional vertigo of vertical semicircular canals. Most serious causes of central vertigo in patients with $p$-DBN can be diagnosed by magnetic resonance imaging of the posterior fossa and craniovertebral junction. However, the peripheral $p$-DBN could be either due to anterior semicircular canal benign paroxysmal positional vertigo (ASC-BPPV) or a recently described apogeotropic variant of posterior semicircular canal BPPV (apo-PSC-BPPV) and the two are almost impossible to differentiate initially. The usual clinical scenario in apo-PSC-BPPV is diagnosing it initially as ASC-BPPV. However, following diagnostic or therapeutic positioning maneuvers for the purported ASC-BPPV, the positional oculomotor pattern changes to an upbeating nystagmus with the reversal in the direction of the torsion as well, localizing it to the contralateral PSC with respect to the ASC initially diagnosed. The initial oculomotor pattern observed on the right Dix-Hallpike test in this patient, of a short latency downbeating left torsional (from the patient's perspective) positional nystagmus suggested a diagnosis of left ASC-BPPV, which was accordingly treated with multiple sessions of reverse Epley maneuvers daily for a week. At the end of the week, a verifying right Dix-Hallpike test elicited an upbeating right torsional (from the patient's perspective) positional nystagmus. It is extremely unlikely that this patient had resolution of her initial left ASC-BPPV with the daily sessions of reverse Epley maneuvers carried over a week and immediately suffered from commoner geotropic variant of the right PSCBPPV (geo-PSC-BPPV). It is plausible to interpret that this patient suffered from the right apo-PSC-BPPV from the very outset, and the reverse Epley maneuver performed for the ostensive left ASC-BPPV led to an intracanal shift of otoconial debris from its
Published online

August 20, 2020
DoI https://doi.org/

$10.1055 / \mathrm{s}-0040-1715532$

ISSN 2581-9607.
(C)2020. Indian Society of Otology.

This is an open access article published by Thieme under the terms of the Creative Commons Attribution-NonDerivative-NonCommercial-License, permitting copying and reproduction so long as the original work is given appropriate credit. Contents may not be used for commercial purposes, or adapted, remixed, transformed or built upon. (https://creativecommons.org/licenses/by-nc-nd/4.0/).

Thieme Medical and Scientific Publishers Pvt. Ltd. A-12, 2nd Floor,

Sector 2, Noida-201301 UP, India 
nonampullary to the ampullary arm resulting in right geo-PSC-BPPV. The reasons why situations like this outwit the clinician resulting in inaccurate localization as well as lateralization is discussed. The patient was successfully treated with right Epley maneuver after transformation to geo-PSC-BPPV and was asymptomatic at follow-up for 4 weeks. A peripheral $p$-DBN with torsional component in any patient with a history of positionally triggered vertigo can be either ASC-BPPV or apo-PSC-BPPV. A very close follow-up at a short interval of time with meticulously executed positional tests is the only definitive way to differentiate the two conditions.

\section{Introduction}

Benign paroxysmal positional vertigo (BPPV) is a mechanical disorder of the membranous labyrinth in which attacks of true rotational vertigo occur when the head changes its position relative to the gravity. The typical situations are laying on the bed, taking lateral recumbent positions, getting up from supine to sitting position, stooping forward (e.g., tying shoelaces), and extending neck (e.g., placing objects on a high shelf). The pathophysiology of BPPV involves inappropriate detachment of the otoconial debris, mainly composed of calcium carbonate derived from the degenerative utricular macula and its sequestration into one of the three semicircular canals (most commonly posterior, less frequently horizontal, and rarely anterior semicircular canal [ASC]). The motion of head in a direction normally moves the endolymph within the semicircular canal aligned in the direction of head movement, bending the cupula to generate an appropriate nerve impulse, which apprises the brain via vestibulo-ocular reflex (VOR), the plane, and the angle the head has moved. The brain reflexely (via VOR) makes corrective eye movements equal in angle, but the opposite direction and the point of fixation thereupon always falls on the fovea centralis.

- Table 1 shows that 56 to 93\% of all patients diagnosed with BPPV at any specialty clinic have posterior semicircular canal BPPV (PSC-BPPV). ${ }^{1-11}$ Owing to its dependent location, the PSC is most frequently involved in BPPV, and the sequestered otoconial debris in canalolithiasis usually gets entrapped in its ampullary arm. The otoconial debris rarely enters the ASC due to its relatively higher location in the membranous labyrinth, and any debris that enters it is liable to get automatically cleared for the same reason.

The diagnosis of BPPV is largely dependent on the elicitation of specific oculomotor patterns (positional nystagmus) by the positional tests, that localize as well as lateralize the affected semicircular canal as per Ewald's laws. ${ }^{12}$ The treatment of BPPV due to canalolithiasis is performed by canalith repositioning maneuvers, innovated for each semicircular canal by the vestibulologists across the globe by conceptualizing the three-dimensional model of the membranous labyrinth. The precise location of otoconia in the semicircular canal is deciphered by observing the oculomotor patterns elicited on the diagnostic positional tests. However, despite the unerring diagnostic precision of the oculomotor patterns obtained by the diagnostic positional tests, some situations outwit the clinician resulting in inaccurate localization as well as lateralization.

In 1995, Agus et a ${ }^{13}$ first observed on positional tests for PSC-BPPV, a reversed oculomotor pattern of positional nystagmus in respect of its vectorial components. It consisted

Table 1 The relative frequencies of different BPPV variants at specialty clinics reported in various studies

\begin{tabular}{|c|c|c|c|c|c|}
\hline Authors & $\begin{array}{l}\text { No. of } \\
\text { patients }\end{array}$ & PSC-BPPV & HSC-BPPV & ASC-BPPV & Multiple canals \\
\hline De la Meilleure et al' 1996 & 287 & $78.05 \%$ & $16.38 \%$ & - & $5.57 \%$ \\
\hline Honrubia et al $\left.\right|^{2} 1999$ & 292 & $85.62 \%$ & $5.14 \%$ & $1.37 \%$ & $7.87 \%$ \\
\hline Macias et al 2000 & 259 & $93.02 \%$ & $1.94 \%$ & - & $5.04 \%$ \\
\hline Korres et al ${ }^{4} 2002$ & 122 & $90.16 \%$ & $8.2 \%$ & $1.64 \%$ & - \\
\hline Sakaida et al 2003 & 50 & $56 \%$ & $38 \%$ & & $6 \%$ \\
\hline Imai et al 2005 & 108 & $64.82 \%$ & $33.33 \%$ & - & $1.85 \%$ \\
\hline Nakayama and Epley 2005 & 833 & $66.39 \%$ & $10.08 \%$ & $2.28 \%$ & $21.25 \%$ \\
\hline Cakir et al ${ }^{8} 2006$ & 169 & $85.21 \%$ & $11.83 \%$ & $1.18 \%$ & $1.78 \%$ \\
\hline Moon et al, ${ }^{9} 2006$ & 1,692 & $60.9 \%$ & $31.9 \%$ & $2.2 \%$ & $5.0 \%$ \\
\hline Jackson et a $\left.\right|^{10} 2007$ & 260 & $66.9 \%$ & $11.9 \%$ & $21.2 \%$ & - \\
\hline Chung et al"1 2009 & 589 & $61.8 \%$ & $35.3 \%$ & $2.9 \%$ & - \\
\hline
\end{tabular}

Abbreviations: ASC-BPPV, anterior semicircular canal benign paroxysmal positional vertigo; HSC-BPPV, horizontal semicircular canal benign paroxysmal positional vertigo; PSC-BPPV, posterior semicircular canal benign paroxysmal positional vertigo. 
of a positional downbeating nystagmus ( $p$-DBN), which was clockwise torsional in the right head-hanging position and counterclockwise torsional in the left head-hanging position (from the examiner's perspective). He attributed this finding to the presence of free-floating otoconial debris in the distal portion of the nonampullary arm of PSC that would result in its ampullopetal shift under the effect of gravitational force at the end of the positioning maneuvers. He also reported that on repeating the diagnostic positional maneuvers (Semont's diagnostic maneuver or Dix-Hallpike maneuver), the oculomotor pattern reversed (upbeating plus counterclockwise torsional in the right head-hanging position and clockwise torsional in the left head-hanging position, from the examiner's perspective). It was hypothesized that at the end of the next "sitting repositioning" maneuver, under the influence of gravitational force, the ampullopetal movement of the otoconial debris shifts it to the juxta-cupular position in the proximal portion of the PSC. In 2012, Vannucchi et al ${ }^{14}$ reported in six patients of positional vertigo: an identical pattern of temporal course and first named it apo-PSC-BPPV. All six patients had an initial oculomotor pattern of torsional DBN on positional tests, that localized the disorder to the ASC, and accordingly, all six were treated with some form of physical therapy for it. However, during regular follow-up over the next few days, all six patients developed a different oculomotor pattern of upbeating positional nystagmus with a reversal in the direction of torsion as well, which localized the disorder to the contralateral PSC. It is extremely unlikely that these six patients had a resolution of their initial ASCBPPV of one side but within a few days developed geo-PSCBPPV (the commoner geotropic variant) of the contralateral side. Ever since the variant apo-PSC-BPPV from Italy has been reported in 23 cases by Califano et al, ${ }^{15} 22$ of whom were successfully treated with a quick liberatory rotation maneuver; Vannucchi et $\mathrm{al}^{16}$ in 16 patients; and AsprellaLibonati and Pecci ${ }^{17}$ in 28 patients described two innovative maneuvers, namely Demi-Semont and 45 degrees forced prolonged positioning, with successful outcomes in 11/16 and $23 / 28$ patients, respectively. Outside Italy there are only two case reports of three patients with apo-PSC-BPPV, two from Illinois, United States, ${ }^{18}$ and one from Cleveland, Ohio, United States. ${ }^{19}$ The apo-PSC-BPPV due to nonampullary arm posterior semicircular canalolithiasis appears to be an important contributor to the expanding realm of the etiology of peripheral $p$-DBN. However, in a study of 77 patients of geoPSC-BPPV, 22 out of those 77, who were successfully treated with Epley maneuver, also exhibited a transient $p$-DBN on a verifying Dix-Hallpike test 1 hour later. The presence of residual debris in the distal nonampullary portion of the PSC was attributed to this finding, and 5 out of the 22 patients (23\%) developed a geo-PSC-BPPV again at a 1 -week follow-up. ${ }^{20}$

We are reporting the case of a 48-year-old female patient with 2 days history of vertigo on lying supine, getting up from supine, and on taking either of the lateral recumbent positions along with a prominent sense of continuous dizziness. She was initially diagnosed as left ASC-BPPV, based on the right Dix-Hallpike maneuver eliciting after a short latency, a downbeating counterclockwise (from the patient's perspective) torsional positional nystagmus. Accordingly, she was treated with sessions of reverse Epley maneuvers for 1 week. At the end of 1 week, the verifying right Dix-Hallpike test elicited an upbeating clockwise (from the patient's perspective) torsional nystagmus suggesting a diagnosis of the typical right geo-PSC-BPPV. Such a temporal course suggests that the patient was suffering from the recently described rare atypical variant of the right apo-PSC-BPPV from the outset, which transformed into the typical geo-PSC-BPPV as a result of the intracanal shift of the otoconial debris from the nonampullary arm of the PSC to the juxta-cupular region in its ampullary arm. To the best of our knowledge, such a rare variant of apo-PSC-BPPV has not been reported from India hitherto.

\section{Case Description}

\section{History}

A 48-year-old female presented on January 23, 2020, with 2 days history of vertigo on lying supine, getting up from supine, and on taking either of the lateral recumbent positions. A prominent sense of dizziness in upright positions instead of true rotational vertigo was also continuously felt by the patient. There was no history of vomiting, diplopia, dysarthria, difficulty in swallowing, hiccups, drooping of upper eyelids, or facial or limb weakness. There was also no history of hypertension, diabetes, coronary artery disease, hypothyroidism, jaundice, craniocervical trauma, cervical radiculopathies, cervical canal stenosis, rheumatoid arthritis, Paget's disease, ankylosing spondylitis, low back dysfunction, spinal cord injuries, or cerebrovascular disease.

\section{Examination}

The general physical examination and vitals of the patient were normal. The screening examination of the cervical spine did not reveal any limitation of movement. The examination of the back region did not reveal kyphoscoliosis. The examination of the lumbosacral spine, including straight leg raising (SLR) test and reverse SLR test, was normal. The neurological examination revealed normal cranial nerve examination; strength was grade 5/5 in all four limbs with normal deep tendon reflexes, and bilateral plantar reflexes were flexor. The examination of the cerebellar system revealed no spontaneous or gaze-evoked nystagmus, and there was no appendicular or axial incoordination. The otoneurological examination revealed normal vertical and horizontal saccadic and smooth pursuit eye movements. The head impulse test was bilaterally normal. Before carrying out positional tests, the presence of spontaneous nystagmus was ruled out by using takeaway Frenzel goggles. ${ }^{21}$ The Dix-Hallpike test was performed as per the published guidelines of the American Academy of Otolaryngology, Head, and Neck Surgery Foundation..$^{22}$ The patient was positioned on examination table in the long-sitting, such that her right side was toward its long free edge, and distance of her bottoms from the head end of the table allowed her head to hang as she was taken to the Dix-Hallpike position. Infrared $\mu$ Frenzel video goggles (model UFG0100BEU, $\mu$ Robotics, Technical Consulting 
\& Research, Inc., Lisbon, Portugal; https://www.roboptics.pt/ index.php/ufrenzel/) were used during the Dix-Hallpike test to eliminate fixation. The patient's head was held with both hands and was rotated 45 degrees to her right in the yaw axis. Thereupon, she was laid in such a manner that her 45 degrees right rotated head was extended 20 degrees on the support of the author's hands to represent the right DixHallpike position. Dix-Hallpike test in the right head-hanging position elicited after a latency of 4 seconds a DBN that was distinctly left torsional (from the patient's perspective) as well persevering till the time positioning was maintained (approximately 2 minutes) (-Video 1 ).

\section{Video 1}

The patient was positioned on the examination table in the long-sitting, such that her right side was towards its long free edge, and the distance of her bottoms from the head end of the table allowed her head to hang as she was taken to the Dix-Hallpike position. The patient's head was held with both hands and was rotated 45 degrees to her right in the yaw plane. Thereupon, she was laid in such a manner that her 45 degrees right rotated head was extended 20 degrees on the support of the author's hands to represent the right Dix-Hallpike position. Right Dix-Hallpike positioning elicited after a latency of $4 \mathrm{sec}-$ onds a downbeating nystagmus that was distinctly left torsional (from the patient's perspective) as well as persevering till the time positioning was maintained (approximately 2 minutes). Online content including video sequences viewable at: https://www.thieme-connect. com/products/ejournals/html/10.1055/s-0040-1715532.

\section{Intervention}

The clinical history, as well as the otoneurologic examination, on January 23, 2020, appeared consistent with the diagnosis of left ASC-BPPV. The patient was initially treated with reverse Epley maneuver ${ }^{2}$ (starting from the uninvolved right head-hanging position). For the reverse Epley maneuver the patient was positioned on examination table in the longsitting such that her left side was toward its free edge. The distance of her bottoms from the head end of the table allowed her head to hang as she was taken to the right DixHallpike position. The patient's head was held with both hands and was rotated 45 degrees to her right in the yaw axis. Thereupon, she was laid in such a manner that her 45 degrees right rotated head was extended 20 degrees on the support of the author's hands to represent the right Dix-Hallpike position (position one). In the right Dix-Hallpike position, the patient's head was maintained for 60 seconds during which a downbeating left torsional (from the patient's perspective) inexhaustible positional nystagmus was observed with concomitant vertigo. Upon completion of 1 minute with 45 degrees of cervical rotation to the presumed contralesional right side, the head was rotated 90 degrees in the rostral-caudal body axis to her left, maintaining its 20 degrees extension (position two). Positioning with 45 degrees of cervical rotation from the sagittal plane to the presumed ipsilesional left side with the neck in 20 degrees of extension was maintained for 1 minute; neither patient complained of vertigo nor had any nystagmus during this period. Thereupon, left lateral recumbent positioning with her nose oriented almost at right angles to the surface of the treatment table was maintained for 1 minute (position three). Lastly, she was instructed to sequentially hang down her lower limbs along the free edge of the examination table, and while maintaining the left rotated head position, she was assisted in taking an upright sitting position completing the reverse Epley maneuver ( $\mathbf{- V i d e o} 2$ ). A total of five reverse Epley maneuvers in a session per day were executed for 1 week and the patient continued to exhibit downbeating left torsional (from the patient's perspective) inexhaustible positional nystagmus in the position one of the maneuvers. During the second day of a treatment session with reverse Epley maneuver, magnetic

\section{Video 2}

For the reverse Epley maneuver, the patient was positioned on the examination table in long-sitting such that her left side was towards its free edge. The distance of her bottoms from the head end of the table allowed her head to hang as she was taken to the Dix-Hallpike position. The patient's head was held with both hands and was rotated 45 degrees to her right in the yaw plane. Thereupon, she was laid in such a manner that her 45 degrees right rotated head was extended 20 degrees on the support of the author's hands to represent the right Dix-Hallpike position (position one). In the right Dix-Hallpike position, the patient's head was maintained for 60 seconds during which a downbeating left torsional (from the patient's perspective) inexhaustible positional nystagmus was observed with concomitant vertigo. Upon completion of 1 minute with 45 degrees of cervical rotation to the presumed contralesional right side, the head was rotated 90 degrees to her left, maintaining its 20 degrees extension (position two). Positioning with 45 degrees of cervical rotation from the sagittal plane to the presumed ipsilesional left side with the neck in 20 degrees of extension was maintained for 1 minute; neither patient complained of vertigo nor had any nystagmus during this period. Thereupon, she was instructed to assume the left lateral recumbent position and rotate her head further leftward so that her nose was positioned almost right-angled with the treatment table (position three). The latter position was maintained for 1 minute. Thereupon, she was instructed to sequentially dangle down her lower limbs along the free edge of the examination table and while maintaining the left rotated head position, she was assisted to assume an upright sitting position completing reverse Epley maneuver. Online content including video sequences viewable at: https://www.thieme-connect. com/products/ejournals/html/10.1055/s-0040-1715532. 
resonance imaging of the brain with dedicated cuts of cerebellum and brainstem, with magnetic resonance screening of the craniovertebral junction was done. The magnetic resonance imaging was normal. Dix-Hallpike test undertaken on day 8 (January 30, 2020), after a week of treatment with daily sessions of reverse Epley maneuvers, elicited after a latency of 2 seconds a crescendo-decrescendo upbeating right torsional (from the patient's perspective) positional nystagmus lasting 45 seconds ( - Video 3 ). Interpreting retrospectively, the correct diagnosis on January 23, 2020, was right apoPSC-BPPV, which emulated contralateral left ASC-BPPV. Treatment of putative left ASC-BPPV with multiple reverse Epley maneuvers for a week (January 23-29, 2020) resulted in an intracanal shift of a possibly jammed otoconial debris from the nonampullary arm of the right PSC into its ampullary arm. The latter resulted in intracanal switch type of right geo-PSC-BPPV. The resultant intracanal switch geo-PSC-BPPV was managed an identical session of five right Epley maneuvers on January 30,2020 . She was instructed to perform the home-based modified self-Epley maneuver five times daily ${ }^{23}$ for the next 2 weeks from January 31, 2020. On February 14,2020 , she was reexamined again and the Dix-Hallpike maneuver in the right head-hanging position for 60 seconds did not elicit any positional nystagmus and she was reported to be free of vertigo since February 9, 2020 ( - Video 4).

\section{Diagnosis}

The aforesaid clinical history, otoneurologic examination, and temporal course of vertigo are consistent with the diagnosis of right apo-PSC-BPPV.

\section{Prognosis and Outcome}

The patient was telephonically questioned weekly regarding the recurrence of rotational vertigo for the next 4 weeks, and it was confirmed that she remained symptom free till then.

\section{Video 3}

The patient was positioned on the examination table in the long-sitting, such that her right side was towards its long free edge, and the distance of her bottoms from the head end of the table allowed her head to hang as she was taken to the Dix-Hallpike position. The patient's head was held with both hands and was rotated 45 degrees to her right in the yaw plane. Thereupon, she was laid in such a manner that her 45 degrees right rotated head was extended 20 degrees on the support of the author's hands to represent the right Dix-Hallpike position. This elicited after a latency of 2 seconds a crescendo-decrescendo upbeating right torsional (from the patient's perspective) positional nystagmus lasting 45 seconds. Online content including video sequences viewable at: https://www.thieme-connect.com/products/ejournals/ html/10.1055/s-0040-1715532.

\section{Video 4}

The patient was positioned on the examination table in the long-sitting, such that her right side was towards its long free edge, and the distance of her bottoms from the head end of the table allowed her head to hang as she was taken to the Dix-Hallpike position. The patient's head was held with both hands and was rotated 45 degrees to her right in the yaw plane. Thereupon, she was laid in such a manner that her 45 degrees right rotated head was extended 20 degrees on the support of the author's hands to represent the right Dix-Hallpike position. The right Dix-Hallpike positioning was maintained for 1 minute and no positional nystagmus was observed. Online content including video sequences viewable at: https://www.thieme-connect.com/products/ejournals/ html/10.1055/s-0040-1715532.

\section{Discussion}

Subjecting a patient with a history of rotational vertigo triggered by changes in the position of head relative to the gravity, to the Dix-Hallpike test, opens a Pandora's box of a diagnostic dilemma if a $p$-DBN is elicited. If such a patient does not have any other central nervous system symptoms or signs, the responsible lesion could be; either serious localizing to the intracranial posterior fossa and craniovertebral junction; or benign due to conditions like ASC-BPPV. A recently described variant of PSC-BPPV due to nonampullary canalolithiasis, called apo-PSC-BPPV, is another diagnostic consideration in such patients. The potentially serious causes of $p$-DBN can be ruled out by obtaining magnetic resonance imaging of the brain, with dedicated views of the posterior fossa structures and the screening of the craniovertebral junction. However, normal magnetic resonance imaging in such patients does not ease out the situation, for the treating otoneurologist. An analysis of the genesis of oculomotor patterns during the right Dix-Hallpike positioning in this patient of right apo-PSC-BPPV, with involved pathophysiologic mechanisms that resulted in positional nystagmus identical in its vectorial components to the left ASC-BPPV is discussed here. In the right Dix-Hallpike test, the otoconial debris located in the ampullary arm of the left ASC shifts ampullofugal, under the effect of gravity triggering excitatory discharges in the left anterior ampullary nerve. However, if otoconial debris is in the nonampullary arm of the right PSC, its ampullopetal movement in the right Dix-Hallpike positioning will trigger inhibitory discharges in the right posterior ampullary nerve. In either of the situations, this will cause upward/clockwise (from patient's perspective) slow phase VOR, due to the combined action of the right (inferior oblique and superior rectus [SR]) and the left (superior oblique and SR). Therefore, the fast-phase VOR which is a refixation saccade (clinically appreciated as positional nystagmus), will be downbeating in 


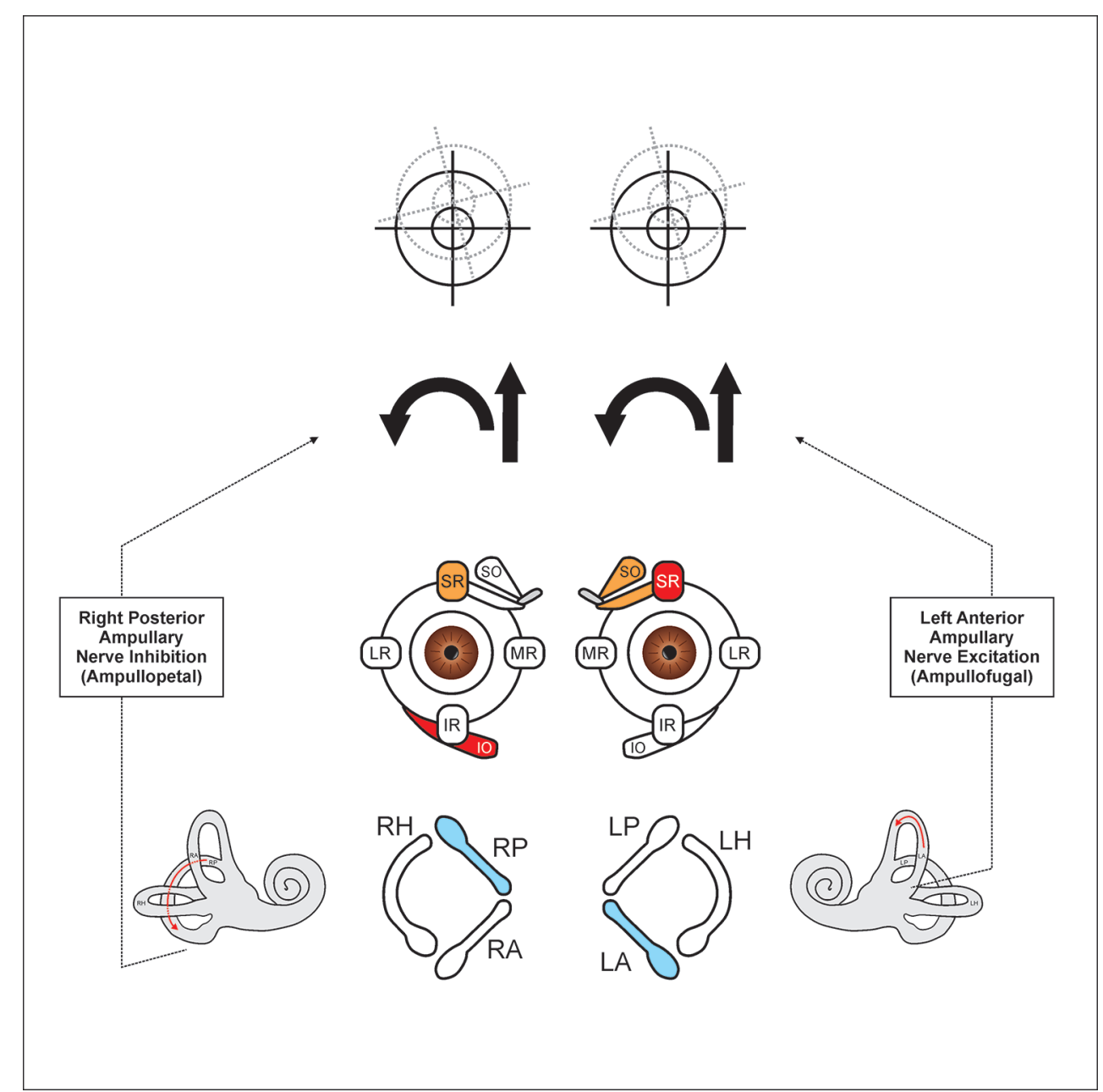

Fig. 1 In the right Dix-Hallpike position, there is an ampullofugal shift of otoconial debris in the ampullary arm of the left anterior semicircular canal, which generates excitatory impulses in the left anterior ampullary nerve. This causes the co-contraction of the right (inferior oblique [IO] and superior rectus [SR]) and the left (superior oblique [SO] and SR). Otoconial debris in the nonampullary arm of the right posterior semicircular canal during the right Dix-Hallpike positioning will result in its ampullopetal shift, which generates inhibitory impulses in the right posterior ampullary nerve, which also causes the co-contraction of the same pair of extraocular muscles. In either of the situations, the generated slow phase VOR is directed upwards (straight arrow) and is clockwise torsional (curved arrow) from the patient's perspective. Therefore, the fast phase VOR (which is appreciated as positional nystagmus) is downbeating and counterclockwise torsional from the patient's perspective. IR, inferior rectus; LA, left anterior semicircular canal; LH, left horizontal semicircular canal; LP, left posterior semicircular canal; LR, lateral rectus; MR, medial rectus; RA, right anterior semicircular canal; RH, right horizontal semicircular canal; RP, right posterior semicircular canal; VOR, vestibulo-ocular reflex.

both eyes with incyclotorsional component in the right eye and excyclotorsional component in the left eye (downwards and counterclockwise from patient's perspective) ( - Fig. 1). It is extremely unlikely that this patient had resolution of her initial left ASC-BPPV with the daily sessions of reverse Epley maneuver carried over a week to be immediately followed by a paroxysm of the commoner geo-PSC-BPPV. It is plausible to interpret that this patient suffered from the right apo-PSC-BPPV from the very outset. Reverse Epley maneuver sessions performed to treat suppositious left ASC-BPPV led to an ampullopetal shift of the otoconial debris jammed in the nonampullary arm of the right PSC to the juxta-cupular location. Thus, this may be looked upon as an intracanal type of canal-switch phenomenon that occurred in the right PSC leading to the changing oculomotor patterns on the right Dix-Hallpike positioning observed on day 1 (January 23, 2020) and day 8 (January 30, 2020).

\section{Conclusion}

In any patient presenting with a history of positionally triggered vertigo, a change in oculomotor patterns elicited on positional tests, suggesting an initial ASC-BPPV (positional downbeating torsional nystagmus) to contralateral geoPSC-BPPV (positional upbeating nystagmus with a reversed vectorial torsional component) over a short period is highly indicative of apo-PSC-BPPV from the very outset. The positional tests or therapeutic maneuvers, which are performed to diagnose/treat the putative condition, lead to an ampullopetal shift of the otoconial debris present in the nonampullary arm of the PSC to the juxta-cupular location. It can be regarded as a form of intracanal switch canalolithiasis within the PSC due to the shift of the otoconial debris from its nonampullary arm to the ampullary arm. The transformation of a nonampullary to the ampullary arm posterior 
semicircular canalolithiasis makes this disorder treatable by the better-established treatment options.

\section{Note}

The views expressed in the submitted article are authors' own and not an official position of the institution to which authors are affiliated.

\section{Conflict of Interest}

None declared.

\section{Acknowledgments}

The authors wish to thank Mr. Renith Kurian, who video recorded the diagnostic and therapeutic maneuvers and precisely captured the nystagmus during the entire diagnostic and treatment period and to Mr. Ashraf Hussain for drawing - Fig. 1 on CorelDraw graphics suite 2019.

\section{References}

1 De la Meilleure G, Dehaene I, Depondt M, Damman W, Crevits L, Vanhooren G. BPPV of the horizontal canal. J Neurol Neurosurg Psychiatry 1996;60(1):68-71

2 Honrubia V, Baloh RW, Harris MR, Jacobson KM. Paroxysmal positional vertigo syndrome. Am J Otol 1999;20(4):465-470

3 Macias JD, Lambert KM, Massingale S, Ellensohn A, Fritz JA. Variables affecting treatment in BPPV. Laryngoscope 2000;110(11):1921-1924

4 Korres S, Balatsouras DG, Kaberos A, Economou C, Kandiloros D, Ferekidis E. Occurrence of semicircular canal involvement in BPPV. Otol Neurotol 2002;23(6):926-932

5 Sakaida M, Takeuchi K, Ishinaga H, Adachi M, Majima Y. Longterm outcome of BPPV. Neurology 2003;60(9):1532-1534

6 Imai T, Ito M, Takeda N, et al. Natural course of the remission of vertigo in patients with BPPV. Neurology 2005;64(5):920-921

7 Nakayama M, Epley JM. BPPV and variants: improved treatment results with automated, nystagmus-based repositioning. Otolaryngol Head Neck Surg 2005;133(1):107-112

8 Cakir BO, Ercan I, Cakir ZA, Civelek S, Sayin I, Turgut S. What is the true incidence of horizontal semicircular canal BPPV. Otolaryngol Head Neck Surg 2006;134(3):451-454

9 Moon SY, Kim JS, Kim BK, et al. Clinical characteristics of BPPV in Korea: a multicenter study. J Korean Med Sci 2006;21(3):539-543

10 Jackson LE, Morgan B, Fletcher JC, Krueger WW. Anterior canal BPPV: an underappreciated entity. Otol Neurotol 2007;28(2):218-222
11 Chung KW, Park KN, Ko MH, et al. Incidence of horizontal canal BPPV as a function of the duration of symptoms. Otol Neurotol 2009;30(2):202-205

12 Ewald JR, Physiologische Untersuchungen Ueber das Endorgan de Nervus Octavus. Wiesbaden, Germany: Bergmann JF Publishers; 1892

13 Agus G, Puxeddu R, Demontis GP, Puxeddu P. Atypical "reversed" paroxysmal positioning nystagmus in BPPV. Acta Otolaryngol Suppl 1995;520(Pt 1) :143-147

14 Vannucchi P, Pecci R, Giannoni B. Posterior semicircular canal benign paroxysmal positional vertigo presenting with torsional downbeating nystagmus: an apogeotropic variant. Int J Otolaryngol 2012;2012:413603

15 Califano L, Salafia F, Mazzone S, Melillo MG, Califano M. Anterior canal BPPV and apogeotropic posterior canal BPPV: two rare forms of vertical canalolithiasis. Acta Otorhinolaryngol Ital 2014;34(3):189-197

16 Vannucchi P, Pecci R, Giannoni B, Di Giustino F, Santimone R, Mengucci A. Apogeotropic posterior semicircular canal benign paroxysmal positional vertigo: some clinical and therapeutic considerations. Audiology Res 2015;5(1):130

17 Asprella-Libonati G, Pecci R. Apogeotropic variant of posterior canal benign paroxysmal positional vertigo. B-ENT 2019;15:119-125

18 Helminski JO. Peripheral downbeat positional nystagmus: apogeotropic posterior canal or anterior canal BPPV. J Neurol Phys Ther 2019;43(Suppl 2):S8-S13

19 Wagner AR. Atypical variants of posterior canal benign paroxysmal positional vertigo after canalith repositioning: a case report. Hearing, Balance and Communication 2019;17(1): 119-126. Doi: 10.1080/21695717.2018.1534471

20 Oh EH, Lee JH, Kim HJ, Choi SY, Choi KD, Choi JH. Incidence and clinical significance of positional downbeat nystagmus in posterior canal benign paroxysmal positional vertigo. J Clin Neurol 2019;15(2):143-148

21 Strupp M, Fischer C, Hanß L, Bayer O. The takeaway Frenzel goggles: a Fresnel-based device. Neurology 2014;83(14): 1241-1245

22 Bhattacharyya N, Gubbels SP, Schwartz SR, et al. Clinical practice guideline: benign paroxysmal positional vertigo (update) executive summary. Otolaryngol Head Neck Surg 2017;156(3):403-416

23 Radtke A, von Brevern M, Tiel-Wilck K, Mainz-Perchalla A, Neuhauser $H$, Lempert T. Self-treatment of benign paroxysmal positional vertigo: Semont maneuver vs Epley procedure. Neurology 2004;63(1):150-152 